\title{
Puerarin restores the autophagic flux to alleviate cadmium-induced endoplasmic reticulum stress in NRK-52E cells
}

\author{
GANG LIU ${ }^{1-3^{*}}$, KANGLEI ZHANG ${ }^{1-3^{*}}$, WENXUAN DONG $^{1-3}$, YUN TAN $^{1-3}$, \\ MENGFEI LONG ${ }^{1-3}$, HUI ZOU ${ }^{1-3}$ and ZONGPING LIU ${ }^{1-3}$ \\ ${ }^{1}$ Department of Clinical Veterinary Medicine, College of Veterinary Medicine; ${ }^{2}$ Jiangsu Co-Innovation Center for Prevention \\ and Control of Important Animal Infectious Diseases and Zoonoses; ${ }^{3}$ Joint International Research Laboratory of Agriculture \\ and Agri-Product Safety of Ministry of Education of China, Yangzhou University, Yangzhou, Jiangsu 225009, P.R. China
}

Received December 4, 2019; Accepted June 1, 2020

DOI: $10.3892 / \mathrm{mmr} .2020 .11301$

\begin{abstract}
Cadmium (Cd) is a heavy metal that can accumulate and cause damage to a variety of tissues and organs. The kidney is the primary target organ for $\mathrm{Cd}$ accumulation and toxic damage. Autophagy, which is a critical intracellular process, serves an important role in maintaining the homeostasis of the intracellular environment. Endoplasmic reticulum stress (ERS) is another key process that functions to promote cell survival or results in cell injury and death. Both autophagy and ERS are associated with oxidative stress; however, the mechanism by which ERS is regulated by autophagy in Cd-induced nephrotoxicity remains unclear. The present study employed a rat NRK-52E cell model, where alterations in cell morphology, density and viability, the accumulation of reactive oxygen species, an increase in malondialdehyde generation and a decrease in antioxidant enzyme activity and apoptosis were induced by $\mathrm{Cd}$ treatment. Cd induced the activation of nuclear factor erythroid 2-related factor 2 (NRF2), an obstruction of autophagic flux and ERS, which were attenuated by puerarin administration. Furthermore, puerarin failed to alleviate ERS following knockdown of autophagy-related protein 7 in NRK-52E cells. Overexpression of Ras-related protein Rab-7, which promotes the fusion of autophagosomes and lysosomes, efficiently reduced ERS. Taken together, these results indicate that puerarin administration restored the autophagic flux to alleviate ERS, via blocking the activation of NRF2.
\end{abstract}

Correspondence to: Dr Zongping Liu, Department of Clinical Veterinary Medicine, College of Veterinary Medicine, Yangzhou University, 12 East Wenhui Road, Yangzhou, Jiangsu 225009, P.R. China

E-mail: liuzongping@yzu.edu.cn

${ }^{*}$ Contributed equally

Key words: cadmium, oxidative stress, puerarin, autophagy, endoplasmic reticulum stress

\section{Introduction}

Cadmium (Cd) is an environmental pollutant and an industrial heavy metal that significantly endangers public health, and exhibits a long biological half-life of 10-30 years (1). Moreover, cigarette smoke and Cd-contaminated food and drinking water are the principal exposure routes for $\mathrm{Cd}$, which is absorbed via the respiratory and digestive tract, respectively, resulting in the accumulation of $\mathrm{Cd}$ in the organism (2). The kidney has been considered to be particularly sensitive to $\mathrm{Cd}(3,4)$, which accumulates in different nephron segments via the blood circulation, and the proximal tubules have been indicated to be more sensitive during the later stage of intoxication (5). A short-term exposure of cells to $\mathrm{Cd}$ has been indicated to cause a destruction of tight and gap junctions, and result in an increase of cell viability without causing cell death $(6,7)$. By contrast, a long-term exposure to $\mathrm{Cd}$ has been revealed to result in a decreased resistance to oxidation and oxidative stress which is reflected by the levels of reactive oxygen species (ROS). ROS are formed as a by-product of the normal metabolism of oxygen, and a notable increase in ROS levels can hinder cell function, leading to mitochondrial damage and ultimately, apoptosis $(8,9)$. Indeed, a previous study has suggested that apoptosis was primarily responsible for Cd-induced cell death (10).

Autophagy is an evolutionarily conserved metabolic process, via which the intracellular components that are enveloped by the autophagy membrane are transported to the lysosome and eventually degraded for recycling (11). Autophagy is a cellular adaptive response, which is regulated by a variety of autophagy-related genes $(12,13)$. In addition, autophagy has been indicated to exert two opposing functions: i) An adaptive mechanism to remove damaged organelles or proteins to maintain cellular homeostasis; and ii) autophagic cell death, which is caused by excessive autophagy (14-16). It has been reported that autophagy promoted cell survival or induced cell death depending on the different growth conditions, cell types and type of stimulus (17). Autophagosomes are spherical structures with double layer membranes serve a key role in autophagy. Accumulation of autophagosomes may be associated with increased autophagosome synthesis or 
obstruction of autophagosome degradation by lysosomes, i.e. blockade of the autophagic flux (12). Autophagosomes are degraded following fusion with lysosomes, a process which is regulated by RAB7 (18). Numerous studies have suggested that autophagy may be induced via an exposure to Cd (19-22). Endoplasmic reticulum (ER) stress (ERS) has been indicated to cause apoptosis in a variety of pathological conditions, including neurodegenerative disorders, infections, drug intoxication, metabolic diseases and heavy metal intoxication (23-25). ER is a network structure, which is connected by membranes, and is primarily responsible for the synthesis of biological macromolecules, such as proteins, lipids and sugars (26). A variety of stimuli, such as oxidative and glycosylation stress, can cause protein unfolding and misfolding, resulting in the accumulation of proteins in the ER lumen, which triggers ERS (27). In vertebrates, ERS is characterized by three different types of protein sensors located at the ER inner membrane, protein kinase $\mathrm{R}$ (PKR)-like ER kinase (PERK), eukaryotic initiation factor $2 \alpha$ (eIF2 $\alpha$ ) and inositol-requiring enzyme 1 (IRE1). ERS has been indicated to promote restoration of ER homeostasis and cell survival, while excessive ERS has been revealed to result in cell injury and death $(28,29)$. Autophagy and ERS are critical mechanisms that are associated with various physiological and pathological processes, such as the physical activity of neurons and chronic obstructive pulmonary disease (26). An interaction between autophagy and ERS has been revealed in previous studies (30-32). A number of studies have reported that NRF2, a transcription factor that regulates the anti-oxidative stress response, is associated with both autophagy and ERS $(33,34)$. Under normal conditions, KEAP1 interacts with NRF2 and forms protein complexes in the cytoplasm, thereby inhibiting the activity of NRF2. However, under oxidative stress NRF2 has been reported to be released from KEAP1 and transferred to the nucleus, where it regulates the expression levels of antioxidant enzyme genes (35). A number of studies have reported that the NRF2/KEAP1 pathway regulates autophagy under oxidative stress (36-38). It has been reported that autophagy may be triggered or regulated by ERS (39). By contrast, another study suggested that autophagy may confer cellular protection via suppressing ERS (40). This discrepancy may depend on the different cell types or stimuli, and the regulatory mechanism is still unclear.

In recent years, extracts from a variety of Chinese medicinal herbs have been widely studied as therapeutic drugs for different diseases. Puerarin $\left(\mathrm{C}_{21} \mathrm{H}_{20} \mathrm{O}_{9}\right)$ is a plant isoflavone, which is extracted from the dried root of the Chinese medicinal herb kudzu. Several studies have reported that puerarin exhibited a wide range of pharmacological effects, such as anti-oxidant, anti-apoptotic and anti-inflammatory functions, decreasing the blood pressure, and improving the microcirculation and neuroprotection (41-43). However, the protective mechanism of puerarin in Cd-induced cytotoxicity is largely unknown, to the best of our knowledge, and requires additional studies. Therefore, in the present study, the immortalized normal rat renal proximal tubular cell line NRK-52E was used as a research model to investigate whether puerarin relieves ERS via regulating autophagy in Cd-induced nephrotoxicity.

\section{Materials and methods}

Chemicals and reagents. All chemicals were of the highest purity gradeavailable.DMEM,Opti-MEM ${ }^{\circledR}$ IReduced-Serum Medium, FBS, trypsin-EDTA, Lipofectamine ${ }^{\circledR} 3000$ Transfection Reagent and Lipofectamine ${ }^{\circledR}$ RNAiMAX Transfection Reagent were purchased from Thermo Fisher Scientific, Inc. Puerarin, cadmium chloride, 2',7'-dichlorofluorescein diacetate (DCFH-DA), DMSO and Cell Counting Kit-8 (CCK-8) were purchased from Merck KGaA. The malondialdehyde (MDA) detection kit and all antioxidant enzyme detection kits, including Glutathione Peroxidase (GSH-px) (cat. no. A005-1-1), Reduced GSH (cat. no. A006-2-1), Superoxide Dismutase (SOD) (cat.no. A001-3-2) and Catalase (CAT) (cat. no. A007-1-1) assay kits, were purchased from Nanjing Jiancheng Bio-Engineering Institute Co., Ltd. The short hairpin RNA (shRNA) targeting autophagy-related protein 7 (ATG7; cat no. RSH046234) and the plasmid encoding the open reading frame (ORF) of Ras-related protein Rab-7 (RAB7; cat. no. Rn25016) were purchased from GeneCopoeia, Inc. The following primary antibodies were used: Microtubule-associated protein 1 light chain $3 \beta$ (LC3B; cat. no. L7543) and p62/sequestosome-1 (SQSTM1; cat. no. P0067), were purchased from Merck KGaA. Binding-immunoglobulin protein (BIP; cat. no. ab227865), CCAAT-enhancer-binding protein homologous protein (CHOP; cat. no. ab11419), nuclear factor erythroid 2-related factor 2 (NRF2; cat. no. ab137550), kelch-like ECH-associated protein 1 (KEAP1; cat. no. ab139729), heme oxygenase-1 (HO-1; cat. no. ab189491), NAD(P)H dehydrogenase [quinone] 1 (NQO1; cat. no. ab80588), superoxide dismutase (SOD) 1 (SOD1; cat. no. ab16831), SOD2 (cat. no. ab13533) and catalase (CAT; cat. no. ab16731) were purchased from Abcam. Phosphorylated (p)-eIF2 $\alpha$ (cat. no. 3398S), activating transcription factor 4 (ATF-4; cat. no. 11815), cleaved caspase-3 (cat. no. 9661), histone H3 (cat. no. 4499S), $\beta$-actin (cat. no. 4970L) and horseradish peroxidase-conjugated goat anti-rabbit (cat. no. 7074) or horse anti-mouse (cat. no. 7076) IgG secondary antibodies were purchased from Cell Signaling Technology, Inc. Bicinchoninic acid (BCA) protein assay kit was purchased from Beyotime Institute of Biotechnology. All other chemicals were purchased from Merck KGaA.

Cell culture. The NRK-52E cell line was cultured in DMEM with $5 \% \mathrm{FBS}$ and $100 \mathrm{U} / \mathrm{ml}$ penicillin-streptomycin at $37^{\circ} \mathrm{C}$ in humidified $5 \% \mathrm{CO}_{2}$ atmosphere. The cells were sub-cultured with trypsin-EDTA digestion, incubated at $37^{\circ} \mathrm{C}$ for $45 \mathrm{sec}$. When the cells reached $75 \%$ confluence, they were used for subsequent experiments.

Cell viability assay. The NRK-52E cells were seeded in 96-well plates at a density of $1 \times 10^{4}$ cells/well. The cells were treated with $0.0,2.5,5.0,10.0,20.0$ and $40.0 \mu \mathrm{M} \mathrm{Cd}$ for $12 \mathrm{~h}$ when the confluence reached $75 \%$, to assess cell viability. Reagent from Cell Counting Kit- 8 was added to each well and incubated in a $37^{\circ} \mathrm{C} \mathrm{CO}_{2}$ incubator for $1-2 \mathrm{~h}$ in the dark, according to the manufacturer's instructions. The optical density was measured using a full-wavelength microplate reader at a wavelength of $450 \mathrm{~nm}$. 
ROS measurement. DCFH-DA was diluted 1:1,000 with serum-free DMEM to a final concentration of $100 \mu \mathrm{M}$. NRK-52E $\left(0.4 \times 10^{6}\right)$ cells were seeded in 6-well plate, treated with $0.0,2.5,5.0,10.0,20.0$ and $40.0 \mu \mathrm{M} \mathrm{Cd}$ in a $37^{\circ} \mathrm{C} \mathrm{CO}_{2}$ incubator for $12 \mathrm{~h}$, then $1.5 \times 10^{6}$ cells were collected and suspended in $1 \mathrm{ml}$ diluted DCFH-DA and incubated in a $37^{\circ} \mathrm{C}$ $\mathrm{CO}_{2}$ incubator for $30 \mathrm{~min}$. The $0 \mu \mathrm{M}$ Cd treated NRK-52E cells suspended in PBS were used as the negative control. The solutions were mixed by inversion every 3-5 min to fully integrate the probe with the cells. The cells were washed three times with PBS to remove excess DCFH-DA, and the fluorescence intensity (FL-1; $530 \mathrm{~nm}$ ) of 10,000 cells was measured using a flow cytometer.

Detection of MDA and antioxidant enzyme activities. The NRK-52E cells were seeded at a density of $6-8 \times 10^{5}$ in $60 \mathrm{~mm}$ dishes and were exposed to $0.0,2.5,5.0,10.0,20.0$ and $40.0 \mu \mathrm{M}$ $\mathrm{Cd}$ in a $37^{\circ} \mathrm{C} \mathrm{CO}_{2}$ incubator for $12 \mathrm{~h}$. The cells were collected and lysed in ice-cold PBS on ice by sonication. The parameters of the sonicator were adjusted to $35 \%$ amplitude, 30 sec sonication and $5 \mathrm{sec}$ sonication with $5 \mathrm{sec}$ between pulses. Following lysis, the supernatant was centrifuged at 15,000 $\mathrm{x}$ g for $5 \mathrm{~min}$ at $4^{\circ} \mathrm{C}$ for subsequent determination. The level of MDA and the activities of GSH-Px, SOD, CAT and GSH were evaluated using commercial kits according to the manufacturer's protocols. BCA protein assay kit was used to quantify the protein concentration of the samples.

Red fluorescent protein (RFP)-LC3 and enhanced green fluorescent protein (EGFP)-RFP-LC3 transfection. RFP-LC3 and EGFP-RFP-LC3 plasmids were gifts from Dr. Lin Wang (College of Animal Science and Veterinary Medicine, Shandong Agricultural University, Tai'an, China). NRK-52E cells $\left(2 \times 10^{5}\right.$ cells/well) were seeded onto sterile coverslips in 24-well plates before treatment. Subsequently, the cells were transfected with RFP-LC3 or EGFP-RFP-LC3 followed by 0,10 and $20 \mu \mathrm{M} \mathrm{Cd}$ treatment in a $37^{\circ} \mathrm{C} \mathrm{CO}_{2}$ incubator for $12 \mathrm{~h}$. Briefly, the cells were cultured in Opti-MEM for $2 \mathrm{~h}$ in a $37^{\circ} \mathrm{C} \mathrm{CO}_{2}$ incubator, followed by culture in transfection medium (Opti-MEM, $50 \mu \mathrm{l}$; plasmid, $0.75 \mu \mathrm{g}$; Lipofectamine ${ }^{\circledR}$ 3000 Reagent, $1.5 \mu \mathrm{l}$; P3000 Reagent, $1 \mu \mathrm{l}$ ) for 3 days in a $37^{\circ} \mathrm{C} \mathrm{CO}_{2}$ incubator according to the manufacturer's protocol. The coverslips were washed in PBS, mounted onto slides and inspected under a 1.4 phase-contrast oil-immersion lens, Leica laser scanning confocal microscope (Leica Microsystems $\mathrm{GmbH}$ ) (magnification, x63).

shRNA and plasmid transfection. NRK-52E cells $\left(2 \times 10^{5}\right.$ cells/well) were seeded in 24 -well plates before transfection. For each well, the cells were transfected with 60 pmol shRNA against ATG7 or $1 \mu \mathrm{g}$ plasmid (ORF Rab7) in a $37^{\circ} \mathrm{C} \mathrm{CO} \mathrm{CO}_{2}$ incubator for $6 \mathrm{~h}$ using Lipofectamine ${ }^{\circledR}$ RNAiMAX or Lipofectamine ${ }^{\circledR} 3000$ Transfection Reagent according to the manufacturer's protocol. Scrambled control (CSHCTR001-nU6; GeneCopoeia, Inc.) and empty control vector (EX-NEG-M39; GeneCopoeia, Inc.) were used as controls. Subsequently, the culture medium was replaced with complete DMEM medium for another $6 \mathrm{~h}$. The cells were subjected to a second transfection, as described above, for $12 \mathrm{~h}$. At $24 \mathrm{~h}$ after transfection, the cells were treated with $20 \mathrm{Cd}$ and/or $100 \mu \mathrm{M}$ puerarin in $37^{\circ} \mathrm{C} \mathrm{CO}_{2}$ incubator for $12 \mathrm{~h}$

Western blot analysis. NRK-52E cells were lysed in RIPA lysis buffer to obtain the total protein extract. Nuclear proteins were obtained using CelLytic ${ }^{\mathrm{TM}}$ NuCLEAR $^{\mathrm{TM}}$ Extraction kit according to the manufacturer's protocol. The BCA protein assay kit was used to quantify the protein concentration. Equal amounts $(20 \mu \mathrm{g})$ of protein lysates were separated via SDS-PAGE on an 8-15\% gel and were transferred to 0.22 or $0.45-\mu \mathrm{m}$ PVDF membranes. The membranes were subsequently blocked for $60 \mathrm{~min}$ with $5 \%$ skimmed milk in TBS $+0.1 \%$ Tween-20 at room temperature. The membranes were then incubated overnight at $4{ }^{\circ} \mathrm{C}$ with the primary antibodies $(1: 1,000)$. Next, the membranes were incubated with horseradish peroxidase-conjugated goat anti-rabbit or horse anti-mouse IgG secondary antibody $(1: 2,000)$ at room temperature for $60 \mathrm{~min}$, followed by incubation with ECL reagent. The level of protein expression was determined via computer-assisted densitometric analysis (GS-800 ${ }^{\mathrm{TM}}$ Densitometer; Quantity One v4.6.6 software; Bio-Rad Laboratories, Inc.). Protein expression was quantified using Image Lab v6.0.1 software (Bio-Rad Laboratories, Inc.). Histone $\mathrm{H} 3$ or $\beta$-actin $(1: 1,000)$ was used as the loading control. Each blot was performed in triplicate.

Statistical analysis. The data were analyzed via one-way ANOVA followed by Scheffe's test using SPSS Statistics v19.0 software (IBM Corp.) and are presented as the mean \pm standard error of the mean of at least three independent experiments. $\mathrm{P}<0.05$ was considered to indicate a statistically significant difference.

\section{Results}

Cd induces oxidative stress. Alterations in morphology and viability are considered to be direct indexes of cell injury (44). Fig. 1A depicts the cell morphology, which was associated with Cd toxicity, as observed under a Nikon ECLIPSE TE200 light microscope (magnification, $\mathrm{x} 20$ ). The control group exhibited confluence, a uniform distribution, and complete morphology without shrinking and swelling. Compared with the control, the cells treated with increasing doses of $\mathrm{Cd}$ exhibited a decreased density, shrinkage and a round shape. Moreover, compared with the control, cell viability was decreased following treatment with 5-40 $\mu \mathrm{M} \mathrm{Cd}$ for $12 \mathrm{~h}$ (Fig. 1B). As illustrated in Fig. 1C, the levels of ROS increased up to 14.96-fold under increasing concentrations of $\mathrm{Cd}$, compared with control cells. As indicated in Fig. 1D, MDA levels increased up to 7.86-fold under increasing concentrations of $\mathrm{Cd}$, compared with control cells. These data indicated that $\mathrm{Cd}$ treatment induced oxidative stress, decreased cell viability and increased cellular damage in NRK-52E cells.

Cd decreases the antioxidant capacity and promotes apoptosis. The activities of antioxidant enzymes and the levels of antioxidants were examined to evaluate the antioxidant status of NRK-52E cells following exposure to various doses of $\mathrm{Cd}$ for $12 \mathrm{~h}$. As demonstrated in Fig. 2, the activities of GSH-Px, SOD and CAT were reduced in the Cd-treated 
A

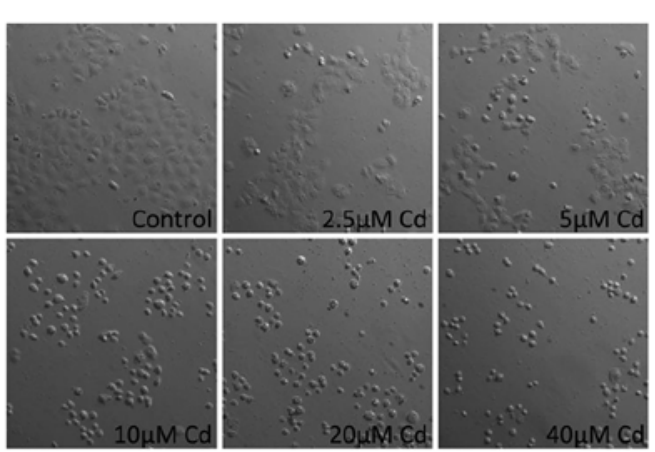

C

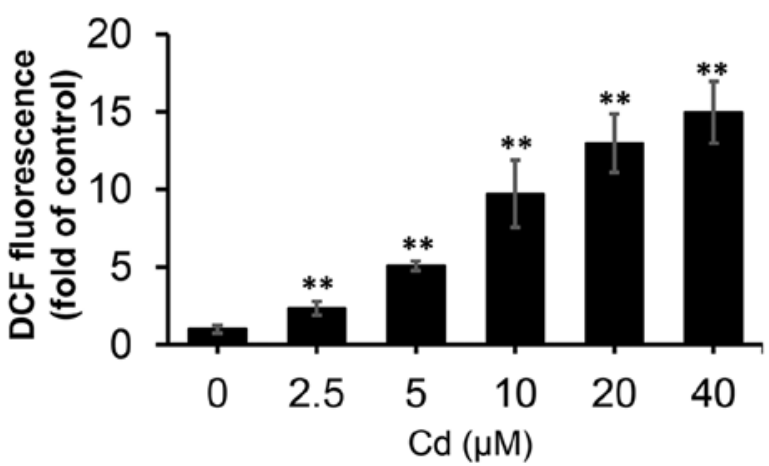

B

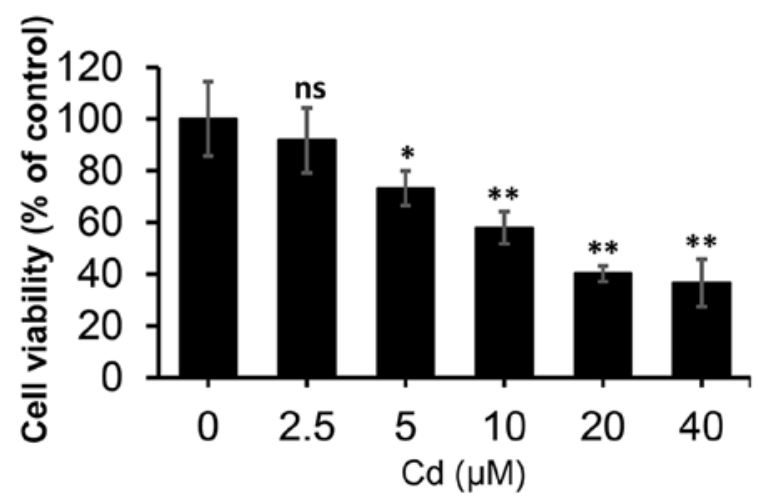

D

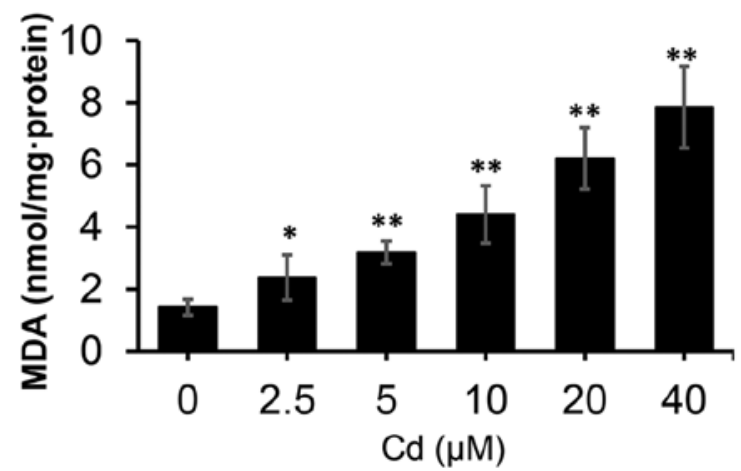

Figure 1. Effect of Cd on the morphology, viability and oxidative stress status of NRK-52E cells. The cells were treated with different concentrations of Cd $(0,2.5,5.0,10,20$ and $40 \mu \mathrm{M})$ for $12 \mathrm{~h}$. (A) Cell morphology was examined under Nikon ECLIPSE TE200 light microscope (magnification, x200). (B) Cell Counting Kit-8 reagent was used to determine cell viability. (C) DCF fluorescence was measured to reflect ROS levels using flow cytometry. (D) MDA levels were measured using a commercial kit. $\mathrm{n}=6$. ${ }^{*} \mathrm{P}<0.05$ and ${ }^{* *} \mathrm{P}<0.01$ vs. $0 \mu \mathrm{M}$ Cd. Cd, cadmium; DCF, dichlorofluorescein; MDA, malondialdehyde; ROS, reactive oxygen species; ns, not significant.

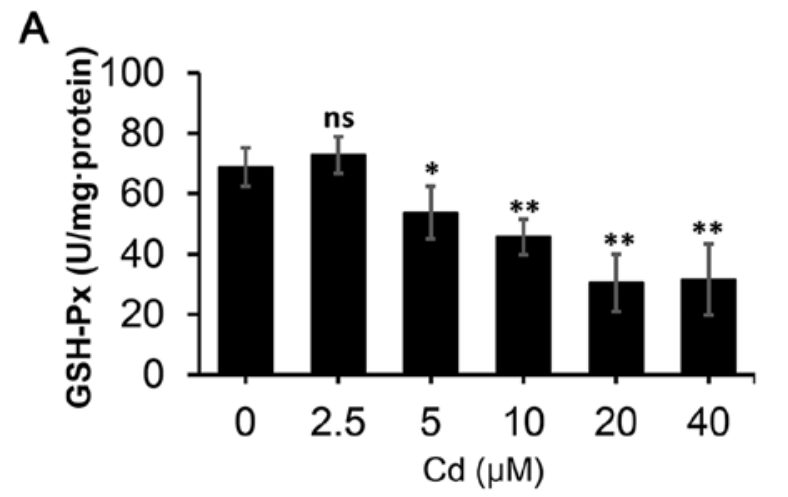

C

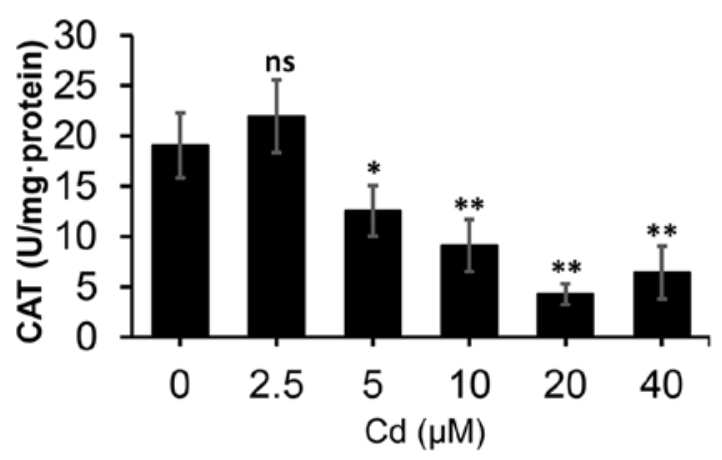

B

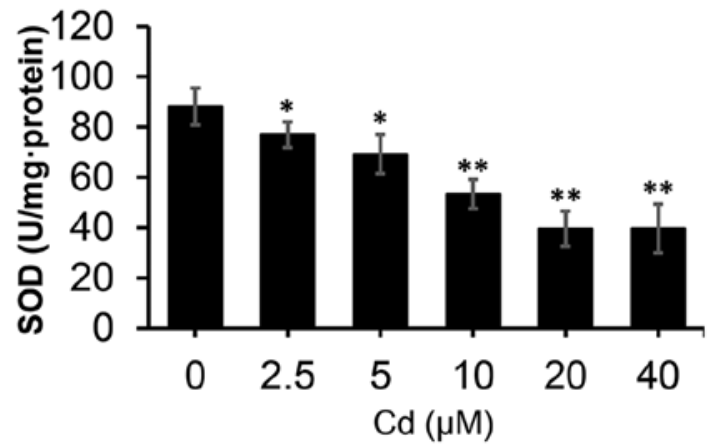

D

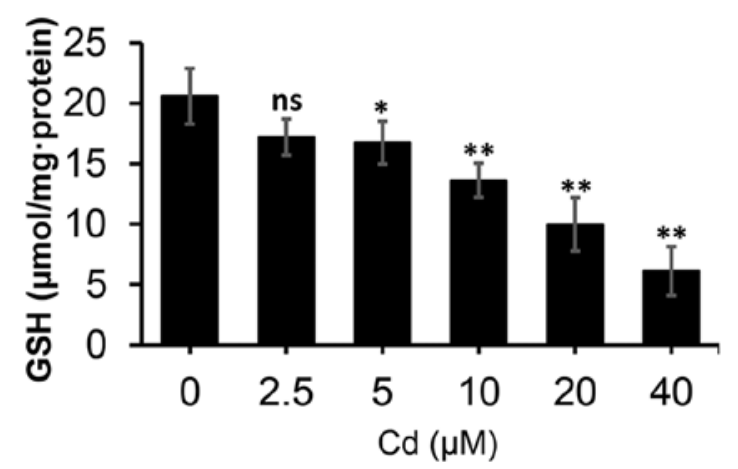

Figure 2. Oxidative stress assays in NRK-52E cells. The cells were treated with different concentrations of Cd $(0,2.5,5.0,10,20$ and $40 \mu \mathrm{M})$ for $12 \mathrm{~h}$, and subsequently the cells were collected to examine the levels of (A) GSH-Px, (B) SOD, (C) CAT and (D) GSH. n=6. ${ }^{*}<<0.05$ and ${ }^{* *} \mathrm{P}<0.01$ vs. $0 \mu \mathrm{M} \mathrm{Cd}$. Cd, cadmium; GSH-Px, glutathione peroxidase; SOD, superoxide dismutase; CAT, catalase; GSH, glutathione; ns, not significant. 

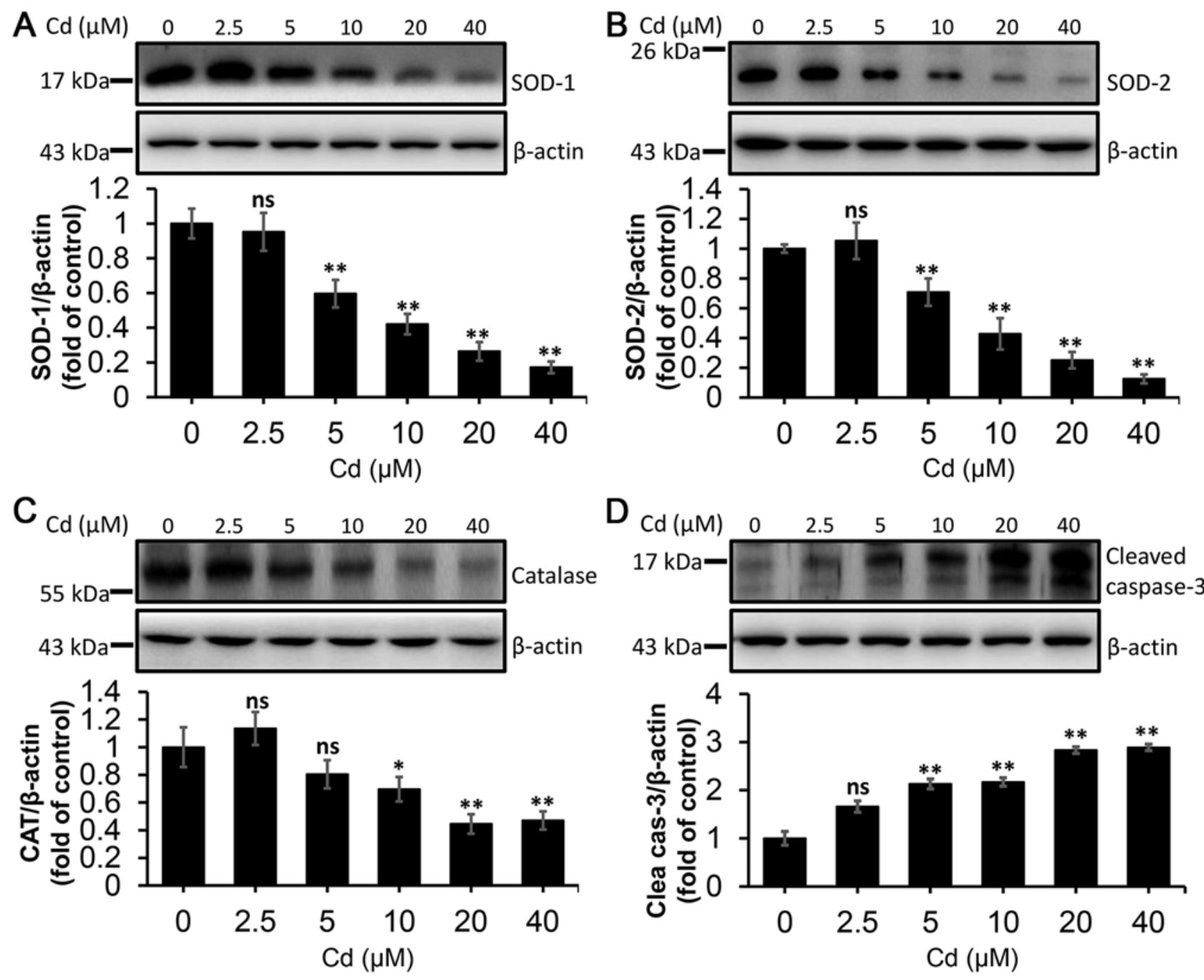

Figure 3. Effect of $\mathrm{Cd}$ on protein levels of antioxidant enzymes and cleaved caspase-3 in NRK-52E cells. The cells were collected and lysed following treatment with a range of Cd concentrations $(0,2.5,5.0,10,20$ and $40 \mu \mathrm{M})$ for $12 \mathrm{~h}$. The protein levels of (A) SOD-1, (B) SOD-2, (C) CAT and (D) cleaved caspase-3 were quantified using densitometry following western blotting. The upper panels depict representative western blotting images and the lower panels indicate the quantitative analysis. $\mathrm{n}=3$. ${ }^{*} \mathrm{P}<0.05$ and ${ }^{* *} \mathrm{P}<0.01$ vs. $0 \mu \mathrm{M} \mathrm{Cd}$. Cd, cadmium; SOD, superoxide dismutase; CAT, catalase; ns, not significant.

groups, exhibiting a reduction up to $0.44,0.45$ and 0.22 -fold, respectively, compared with the control. Moreover, a decrease in GSH levels up to 0.59 -fold compared with the control was observed. The data illustrated in Fig. 3 indicate that $\mathrm{Cd}$ at $5-40 \mu \mathrm{M}$ reduced the protein levels of SOD1 and SOD2, increased the protein level of cleaved caspase-3, and $\mathrm{Cd}$ at $10-40 \mu \mathrm{M}$ decreased the protein levels of CAT, resulting in oxidative stress. These data revealed that $\mathrm{Cd}$ treatment resulted in a decreased antioxidant capacity and increased apoptosis in NRK-52E cells.

Cd inhibits the autophagic flux and promotes ERS. Cd treatment was indicated to induce ROS accumulation, which has been associated with autophagy and ERS; however, the regulatory mechanism between them is controversial (45). To examine this mechanism, the effect of $\mathrm{Cd}$ on autophagy and ERS was assessed. Firstly, the number of RFP-LC3 puncta was observed after transient transfection of the RFP-LC3 plasmid into NRK-52E cells. As illustrated in Fig. 4A, compared with the control group, the number of RFP-LC3 puncta in the $\mathrm{Cd}$-treated group was increased, indicating an accumulation of autophagosomes, which is a key element of the autophagy process (11). To further clarify the cause of autophagosome accumulation, the EGFP-RFP-LC3 plasmid was transfected into NRK-52E cells to detect the autophagic flux. Under normal conditions, the LC3-II-positive autophagosomes are labeled with yellow color (GFP and RFP signals), while autophagosomes are presented as red puncta only after fusion with lysosomes, as GFP is sensitive to the low lysosomal $\mathrm{pH}$ and is rapidly quenched (46). The data in Fig. 4B indicate that, compared with the control group, the accumulation of yellow puncta increased, whereas the accumulation of red puncta decreased after exposure to $\mathrm{Cd}$, revealing that the autophagic flux was blocked. Subsequently, the protein levels of LC3-II and p62 were detected (Fig. 5A and B). The levels of both proteins increased in the $20 \mu \mathrm{M} \mathrm{Cd}$ treatment group, which further suggested that the autophagic flux was inhibited by $\mathrm{Cd}$ treatment. The expression levels of BIP, p-eIF2 $\alpha$, ATF-4 and $\mathrm{CHOP}$ were detected via western blotting to examine Cd-induced ERS. As presented in Fig. 5C-F, the levels of these proteins increased in a dose-dependent manner after treatment with 10 and $20 \mu \mathrm{M} \mathrm{Cd}$ compared with the control group. 

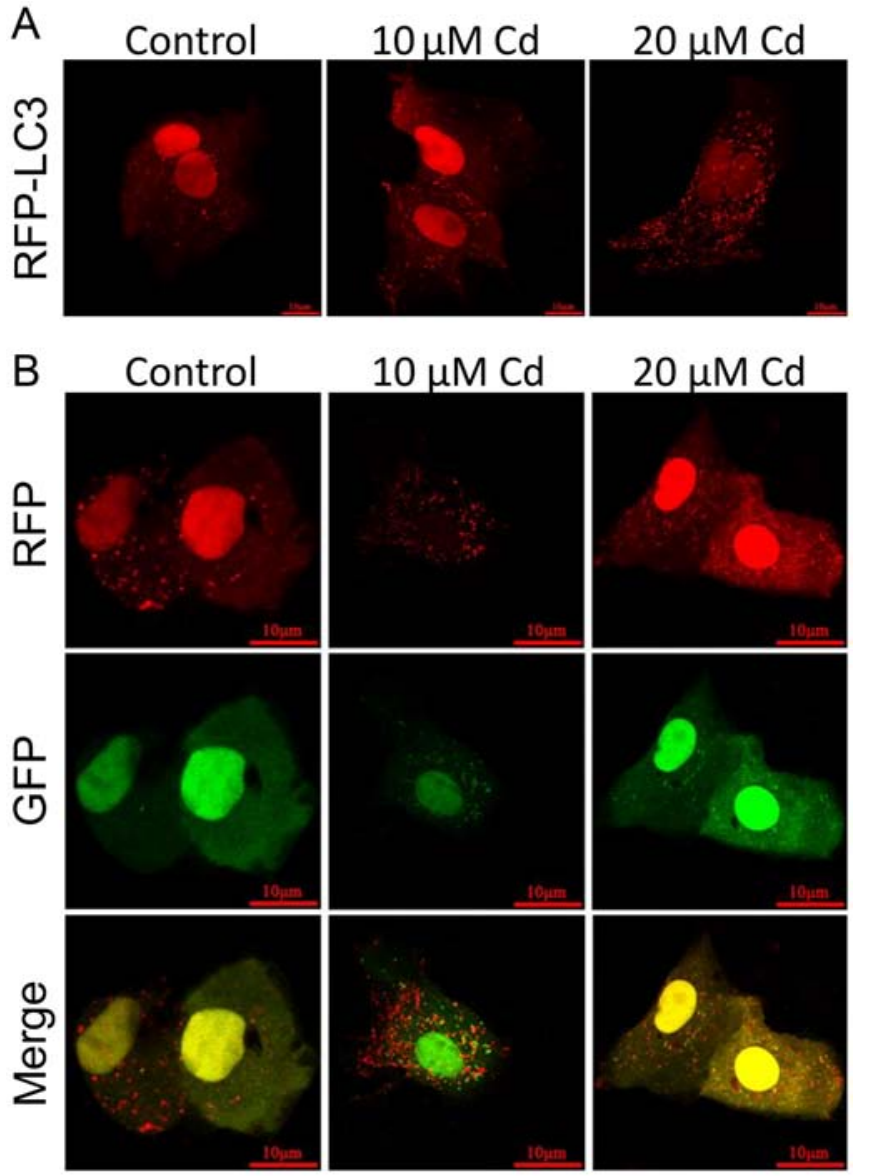

Figure 4. Obstruction of autophagic flux is observed in Cd-treated NRK-52E cells. The cells were transiently transfected with (A) RFP-LC3 and (B) GFP-RFP-LC3 plasmid, and they were subsequently treated with 10 or $20 \mu \mathrm{M} \mathrm{Cd}$ for $12 \mathrm{~h}$. Puncta with both green and red fluorescence (indicated as yellow), or exhibiting only red fluorescence are illustrated in the representative confocal images (magnification, x630). RFP, red fluorescent protein; EGFP, enhanced green fluorescent protein; LC3, microtubule-associated protein 1 light chain $3 ; \mathrm{Cd}$, cadmium

Collectively, these data demonstrated that the autophagic flux was impaired and ERS was induced by $\mathrm{Cd}$ treatment in NRK-52E cells.

Puerarin prevents Cd-induced activation of the NRF2/KEAPI pathway. Western blotting analysis was used to determine the effect of puerarin on the NRF2/KEAP1 pathway in the current study. The results indicated that puerarin inhibited $\mathrm{Cd}$-induced NRF2 translocation into the nucleus (Fig. 6A). Consistently, puerarin attenuated the Cd-mediated decrease in KEAP1 in the cytoplasm, as depicted in Fig. 6B. Moreover, the expression levels of NRF2/KEAP1 downstream proteins were examined via western blotting. The results indicated that the $\mathrm{Cd}$-induced increase in HO-1 and NQO1 levels were prevented via puerarin administration (Fig. 6C and D). These data suggested that Cd-induced activation of the NRF2/KEAP1 pathway may be inhibited via puerarin administration.

Puerarin restores the autophagic flux and alleviates ERS. The results illustrated in Fig. 6 suggested that the NRF2/KEAP1 pathway was regulated by puerarin. NRF2 is a key regulator of autophagy that is associated with ERS (33). In the current study, the effect of puerarin on autophagy and ERS was examined. Consistently with previous findings (47), puerarin aided in the restoration of the autophagic flux following $\mathrm{Cd}$ exposure (Fig. 7A and B). Moreover, the levels of BIP, p-eIF2 $\alpha$, ATF-4 and $\mathrm{CHOP}$ were decreased in the $\mathrm{Cd}$ and puerarin-treated group compared with those in the Cd-treated group, indicating that $\mathrm{Cd}$-induced ERS was alleviated by puerarin (Fig. 7C-F). Furthermore, puerarin alleviated the oxidative stress and the decrease in the antioxidant capacity and cell viability induced by $\mathrm{Cd}$ treatment (Fig. S1). Subsequently, it was examined whether puerarin-induced autophagy is involved in ERS attenuation. ATG7 shRNA was used to construct an autophagy-deficient cell model, and ATG7 protein level was determined in ATG7-deficient NRK-52E cells. The ATG7 level in cells treated with ATG7 shRNA decreased by $65 \%$ (Fig. S2). In the autophagy-deficient cells, puerarin co-treatment was not efficient in decreasing the levels of BIP, p-eIF2A, ATF-4 and CHOP that were induced by Cd (Fig. 8A-D), which indicated that puerarin cannot alleviate the Cd-induced ERS in the absence of autophagy. In conclusion, ERS attenuation following exposure to $\mathrm{Cd}$ was dependent on the restored autophagic flux, which was induced by puerarin. In addition, ATG7 knockdown further exacerbated Cd-induced oxidative stress and the decreasing of antioxidant capacity and cell viability (Fig. S3). These data indicated that puerarin alleviated the Cd-induced ERS primarily via autophagy regulation in NRK-52E cells.

RAB7-induced autophagic flux restoration is involved in alleviating ERS. In cells overexpressing RAB7 (Fig. S4), the Cd-induced blockade of the fusion between autophagosomes and lysosomes was restored (Fig. S5). Moreover, the levels of BIP, p-eIF $2 \alpha$, ATF-4 and CHOP, which were upregulated by $\mathrm{Cd}$, were downregulated in RAB7-overexpressing cells, which indicated that ERS was alleviated by RAB7 overexpression (Fig. 9A-D). In addition, RAB7 overexpression exhibited a positive effect on alleviating oxidative stress, and enhancing antioxidant capacity and cell viability (Fig. S6), which is similar to puerarin (Fig. S1). Additionally, Cd-induced activation of p38 and JNK suppression of ERK were reversed by puerarin administration (Fig. S7). Taken together, these data demonstrated that the restoration of the autophagic flux alleviated ERS, which was indicated to serve a critical role in puerarin-mediated protection against cell injury following exposure to $\mathrm{Cd}$.

\section{Discussion}

In the present study, the effect of autophagy on ERS in Cd-mediated nephrotoxicity was investigated using an NRK-52E cell model. Firstly, it was demonstrated that $\mathrm{Cd}$ treatment induced oxidative damage and apoptosis in NRK-52E cells in a dose-dependent manner. Secondly, Cd was indicated to inhibit the autophagic flux and promote ERS. Thirdly, puerarin was revealed to restore the autophagic flux, which was induced by $\mathrm{Cd}$, and alleviate ERS; however, this effect was not observed in the absence of autophagy in ATG7-deficient NRK-52E cells. Lastly, the NRF2/KEAP1 pathway was indicated to be involved in the process of autophagic flux restoration that prevented ERS in NRK-52E 

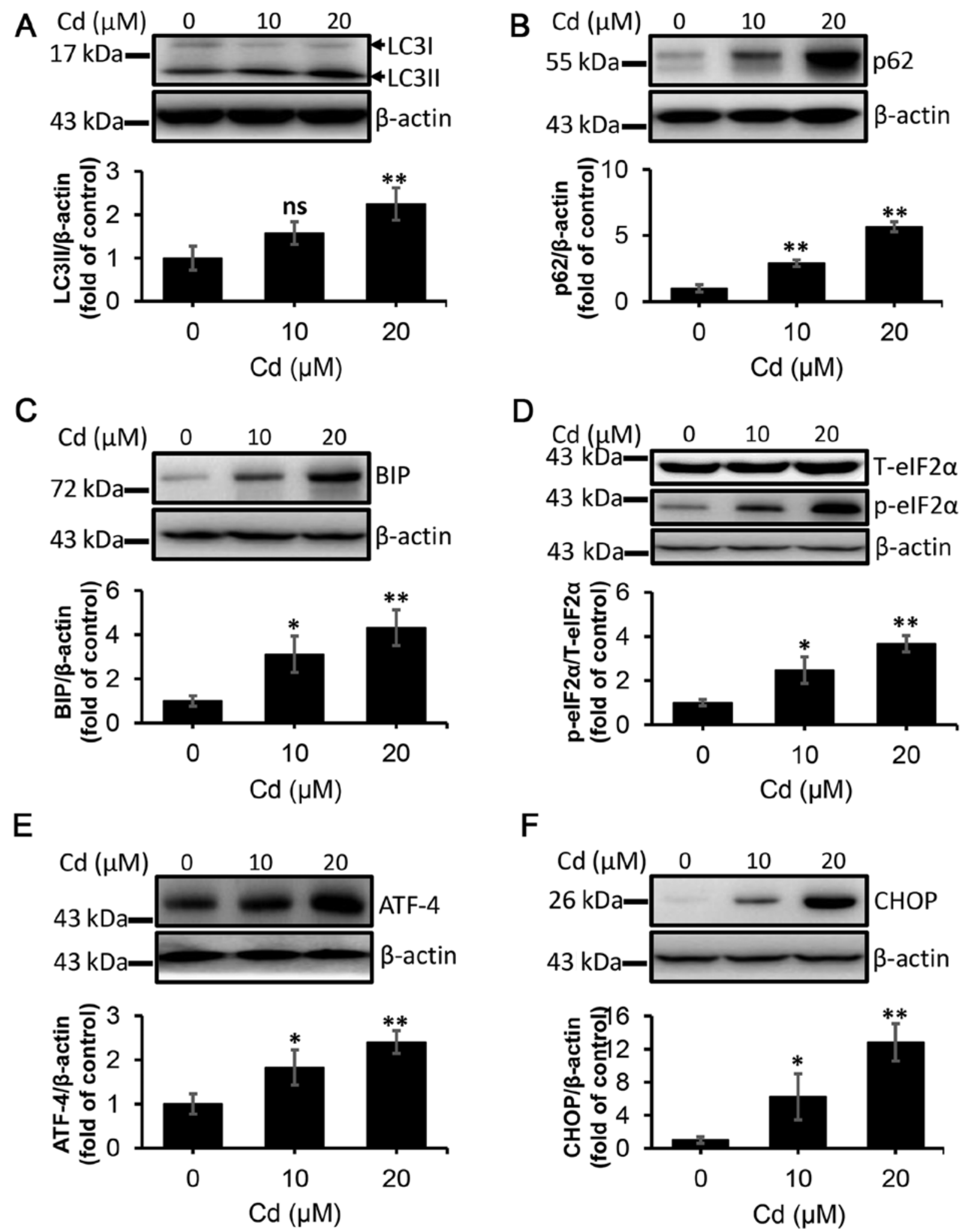

F
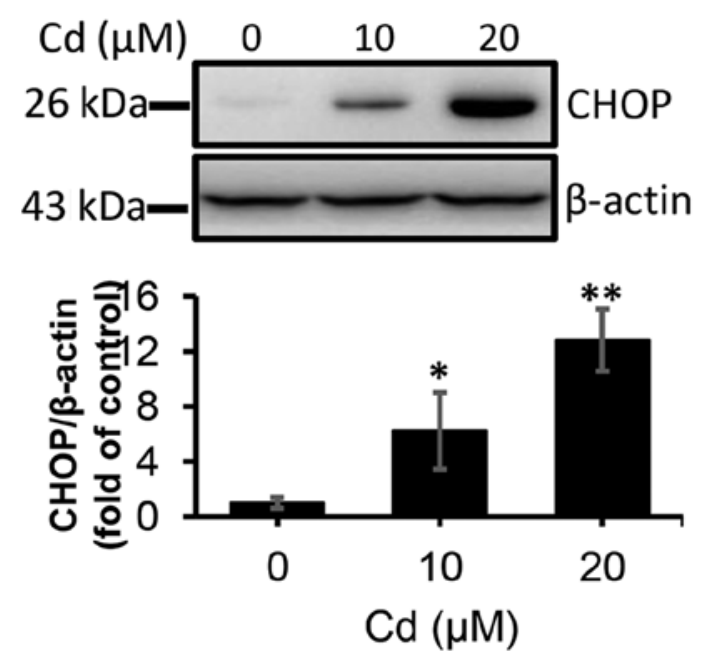

Figure 5. Cd induces autophagic flux blockade and endoplasmic reticulum stress in NRK-52E cells. The cells were collected and lysed following treatment with 10 and $20 \mu \mathrm{M} \mathrm{Cd}$ for $12 \mathrm{~h}$. The protein levels of (A) LC3II, (B) p62, (C) BIP, (D) p-eIF2 $\alpha$, (E) ATF-4 and (F) CHOP were quantified using densitometry following western blotting. $\mathrm{n}=3$. ${ }^{*} \mathrm{P}<0.05$ and ${ }^{* *} \mathrm{P}<0.01$ vs. $0 \mu \mathrm{M} \mathrm{Cd}$. Cd, cadmium; LC3, microtubule-associated protein 1 light chain 3; BIP, binding-immunoglobulin protein; p-eIF2 $\alpha$, phosphorylated eukaryotic initiation factor $2 \alpha$; T-eIF2 $\alpha$, total eukaryotic initiation factor $2 \alpha$; ATF-4, activating transcription factor 4; CHOP, CCAAT-enhancer-binding protein homologous protein; ns, not significant.

cells following exposure to Cd. Collectively, the results of the current study indicated that Cd-induced nephrotoxicity was attenuated by puerarin, which inhibited the NRF2/KEAP1 pathway to restore the autophagic flux and alleviate ERS.
Autophagy and apoptosis are two distinct physiological responses of cells, which regulate cell survival and death after stimulation of cells by different signals, and have been indicated to be associated with ERS and be involved in several 

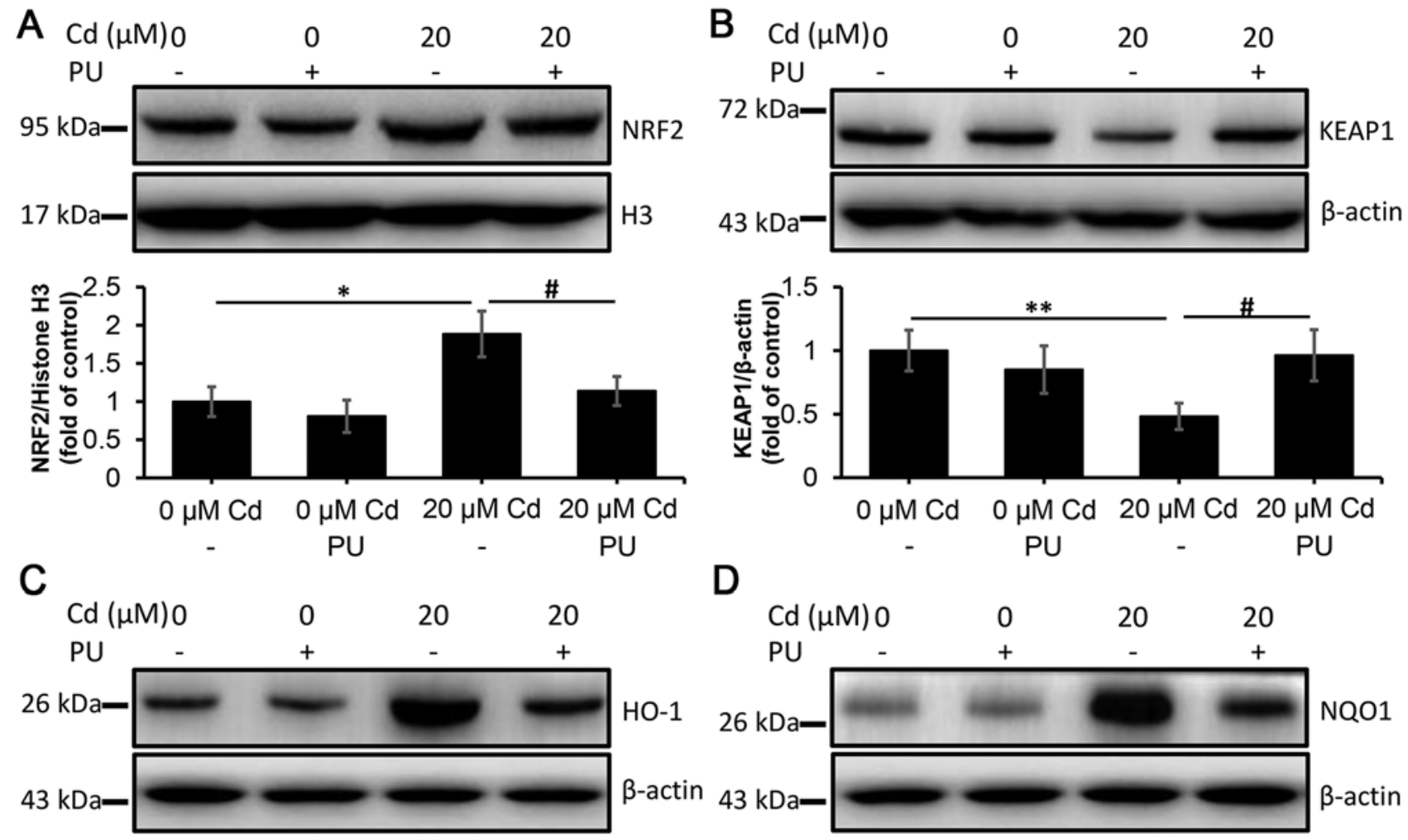

D
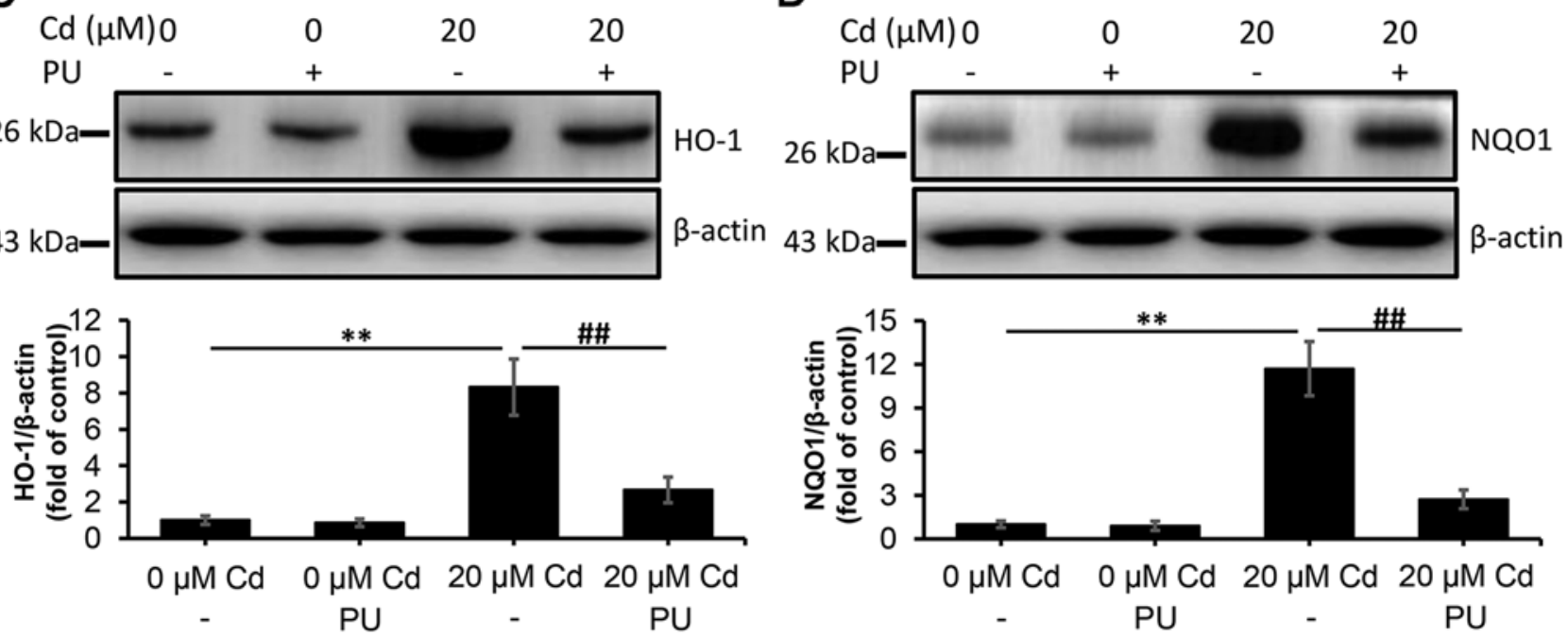

Figure 6. Inhibitory effect of PU on the Cd-induced NRF2/KEAP1 pathway in NRK-52E cells. The cells were treated with $20 \mu \mathrm{M} \mathrm{Cd}$ in the absence or presence of $100 \mu \mathrm{M}$ PU for $12 \mathrm{~h}$, and they were subsequently collected and lysed. The protein levels of (A) nuclear NRF2, (B) KEAP1, (C) HO-1 and (D) NQO1 in total cell lysates were quantified using densitometry following western blotting. The upper panels depict representative western blotting images and the lower panels indicate the quantitative analysis. $\mathrm{n}=3$. ${ }^{*} \mathrm{P}<0.05$ and ${ }^{* *} \mathrm{P}<0.01$ vs. $0 \mu \mathrm{M} \mathrm{Cd} ;{ }^{*} \mathrm{P}<0.05$ and ${ }^{\# \#} \mathrm{P}<0.01$ vs. $20 \mu \mathrm{M}$ Cd and puerarin co-treatment. Cd, cadmium; PU, puerarin; NRF2, nuclear factor erythroid 2-related factor 2; KEAP1, kelch-like ECH-associated protein 1; HO-1, heme oxygenase-1; NQO1, NAD(P)H dehydrogenase [quinone] $1 ; \mathrm{H} 3$, histone $\mathrm{H} 3$.

kidney diseases, such as diabetes, ischemia-reperfusion injury and kidney stones (48). ERS has been revealed to exhibit a dual function by promoting cell survival or apoptosis, and may be associated with autophagy under a variety of pathological conditions (49). Autophagy has been reported to serve a cytoprotective role in acute kidney injury, as blockade of the autophagic flux has been indicated to deteriorate the pathological process of acute kidney injury (50). Autophagy and ERS have been indicated to interact and are implicated in numerous pathological processes, including neurodegenerative disorder, cancer, diabetic nephropathy and renal fibrosis $(30,48,51)$. However, the interaction between autophagy and ERS requires additional elucidation. The present study explored whether a crosstalk between ERS and autophagy inhibition in Cd-treated NRK-52E cells exists, and determined the implicated mechanism. Moreover, the results of the current study provided insight into the signaling mechanisms via which puerarin administration restores the autophagic flux to alleviate ERS during Cd-induced nephrotoxicity.
Both apoptosis and autophagy have been associated with Cd-induced nephrotoxicity $(20,52)$. Moreover, ERS has been indicated to promote renal disease via inducing apoptosis (52). All these processes are closely associated with oxidative stress, which has been reported to be a principal mechanism of Cd-induced toxicity $(53,54)$. The results of the present study indicated that both ROS and MDA levels were increased in NRK-52E cells following treatment with 2.5-40 $\mu \mathrm{M} \mathrm{Cd}$. On the contrary, cell viability, GSH and the levels of antioxidant enzymes were decreased following treatment with 5-40 $\mu \mathrm{M} \mathrm{Cd}$. Moreover, the levels of cleaved caspase-3, which is a marker of apoptosis, were increased in Cd-treated cells. These results are consistent with that of previous reports $(55,56)$ and revealed that $\mathrm{Cd}$ induced oxidative stress and apoptosis in NRK-52E cells. Kidney injury is induced following chronic accumulation of $\mathrm{Cd}$, and this process may last for decades (57). It would be unrealistic to treat the cells with the same $\mathrm{Cd}$ concentration $(0.22-1.03 \mu \mathrm{M})$ as that in blood of patients with renal disease $(58,59)$, which would not be efficient in inducing renal 

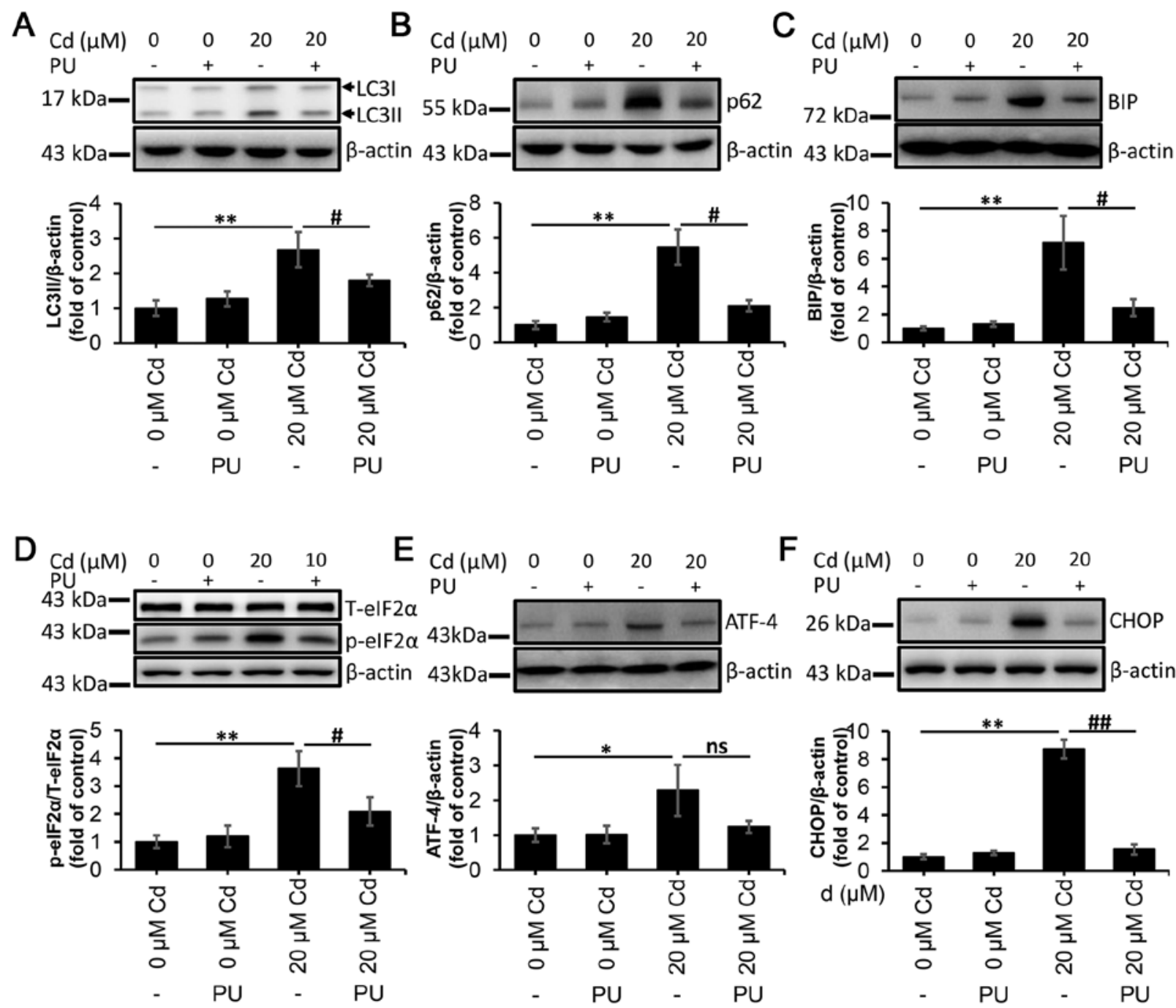

Figure 7. PU restores the autophagic flux and alleviates endoplasmic reticulum stress. The cells were treated with $20 \mu \mathrm{M}$ Cd in the absence or presence of $100 \mu \mathrm{M}$ PU for $12 \mathrm{~h}$, and they were subsequently collected and lysed. The protein levels of (A) LC3II, (B) p62, (C) BIP, (D) p-eIF2 $\alpha$, (E) ATF-4 and (F) CHOP were quantified using densitometry. $\mathrm{n}=3$. ${ }^{*} \mathrm{P}<0.05$ and ${ }^{* *} \mathrm{P}<0.01$ vs. $0 \mu \mathrm{M} \mathrm{Cd}$; ${ }^{\#} \mathrm{P}<0.05$ and ${ }^{\# \#} \mathrm{P}<0.01$ vs. $20 \mu \mathrm{M} \mathrm{Cd}$ and puerarin co-treatment. Cd, cadmium; $\mathrm{PU}$, puerarin; LC3, microtubule-associated protein 1 light chain 3; BIP, binding-immunoglobulin protein; p-eIF2 $\alpha$, phosphorylated eukaryotic initiation factor $2 \alpha$; T-eIF $2 \alpha$, total eukaryotic initiation factor $2 \alpha$; ATF-4, activating transcription factor 4; CHOP, CCAAT-enhancer-binding protein homologous protein; ns, not significant.

cell injury in the limited time of the in vitro study. Therefore, the doses of Cd that were used to treat NRK-52E cells in the current study were higher than the concentration of free $\mathrm{Cd}$ in blood (0.22-1.03 $\mu \mathrm{M})$ and locally in the kidney, in order to simulate renal cell injury, which is induced following decades of $\mathrm{Cd}$ accumulation, and affect the associated mechanisms, such as oxidative stress, organelle damage and apoptosis.

In the present study, ERS was activated and the autophagic flux was inhibited in NRK-52E cells treated with 10 and $20 \mu \mathrm{M} \mathrm{Cd}$. However, the effect of $\mathrm{Cd}$ on autophagy may vary in different cell types. In addition to proximal tubular cells, renal mesangial cells may also be affected by $\mathrm{Cd}$-induced renal injury. Fujishiro et al (20), reported a protective effect of $\mathrm{Cd}$-induced autophagy in rat renal mesangial cells, which is contradictory to the results of the current study. Fujishiro et al (20), used cells that were cultured in medium with $0.2 \%$ FBS for $48 \mathrm{~h}$ before transfer to serum-free medium with $\mathrm{Cd}$. This prolonged serum starvation may have induced additional effects on autophagy, especially in the presence of other stimuli. Moreover, it was suggested that autophagy was primarily regulated by JNK-mediated signaling in rat renal mesangial cells exposed to Cd (16). However, another study has revealed that in addition to JNK, the ERK and p38 pathways are also involved in Cd-induced mouse proximal tubular cell injury (60). Therefore, in the current study, activation of p38, but not JNK, and the ERS-induced inhibition of the autophagic flux mediated the effect of $\mathrm{Cd}$ on autophagy.

Previous studies have indicated that the NRF2/KEAP1 axis is an important endogenous antioxidant signaling pathway $(35,61)$. NRF2 has been reported to be activated following translocation to the nucleus, where it has been indicated to interact with the antioxidant response element (ARE) to regulate the transcription and expression of antioxidant enzymes, thereby enhancing the resistance to oxidative stress (56). In the present study, the Cd-induced nuclear accumulation of NRF2 was inhibited by puerarin. Moreover, the expression levels of KEAP1, which is an inhibitory receptor of NRF2, and two downstream targets of NRF2, HO-1 and 

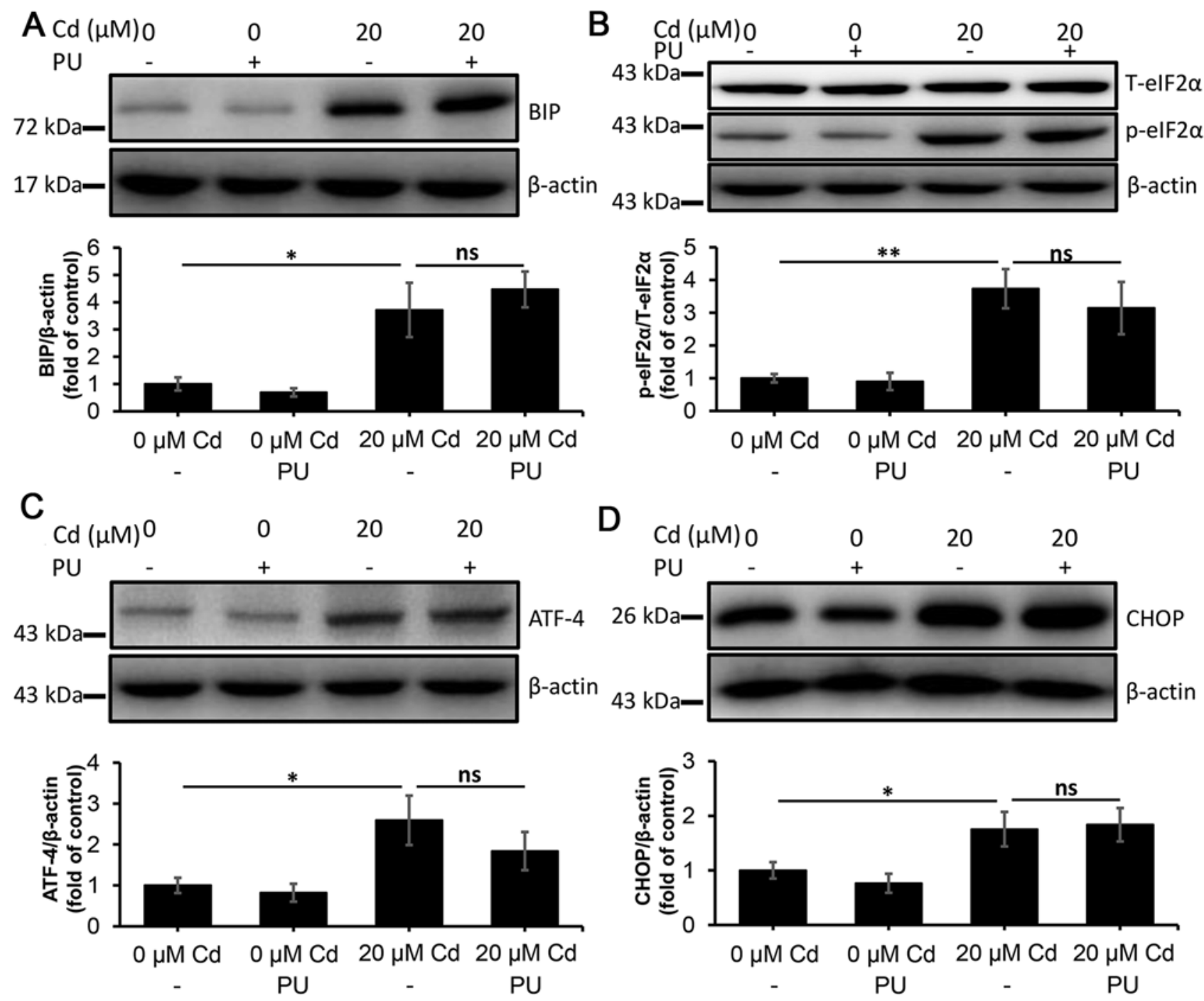

Figure 8. PU fails to alleviate endoplasmic reticulum stress in NRK-52E cells following ATG7 knockdown. The cells were transfected with a short hairpin RNA targeting ATG7, and they were subsequently treated with $20 \mu \mathrm{M}$ Cd in the absence or presence of $100 \mu \mathrm{M}$ PU for $12 \mathrm{~h}$. The protein levels of (A) BIP, (B) p-eIF $2 \alpha$, (C) ATF-4 and (D) CHOP were quantified using densitometry following western blotting. $\mathrm{n}=3$. ${ }^{*} \mathrm{P}<0.05$ and ${ }^{* *} \mathrm{P}<0.01$ vs. $0 \mu \mathrm{M}$ Cd. Cd, cadmium; PU, puerarin; ATG7, autophagy-related protein 7; BIP, binding-immunoglobulin protein; p-eIF2 $\alpha$, phosphorylated eukaryotic initiation factor $2 \alpha$; T-eIF2 $\alpha$, total eukaryotic initiation factor $2 \alpha$; ATF-4, activating transcription factor 4; CHOP, CCAAT-enhancer-binding protein homologous protein; ns, not significant.

NQO1, were detected via western blotting in the current study to indirectly assess the activation status of NRF2. The results indicated that compared with the Cd group, puerarin treatment ablated the downregulation of KEAP1 and the upregulation of HO-1 and NQO1 protein levels. Activation of NRF2 has been indicated to be associated with a series of pathological and physiological processes including respiratory distress syndrome, neurodegeneration, and carcinogenesis and tumor development $(62,63)$, and participates in renal pathology (64). Puerarin has been revealed to protect against $\mathrm{CCl}_{4}$-induced oxidative stress and inflammation via inhibiting the ERK/NRF2/ARE pathway in rat kidneys (65). In addition, puerarin treatment has been indicated to enhance the mRNA expression levels of anti-oxidant enzymes, such as NRF2, HO-1 and SOD2 (66). As oxidative stress and ERS have been indicated to positively regulate the NRF2 signaling pathway (67), they may account for the upregulation of NRF2 in the present study. However, since ERS has not been reported to inhibit the NRF2 signaling pathway, to the best of our knowledge, puerarin-induced NRF2 inhibition may be attributed to the antioxidant capacity of puerarin.

A crosstalk between NRF2 and autophagy under stress conditions has been reported in previous studies $(36,68)$. In addition, both persistent ERS and the inhibition of autophagic flux have been indicated to mediate cell injury, and although ERS may result in autophagy (69), the effect of autophagic flux on ERS is largely unknown. Therefore, the autophagic flux was examined in the NRK-52E cell model in the current study. The blockade of the autophagic flux, which was induced by $\mathrm{Cd}$, was indicated to be attenuated by puerarin. Moreover, Cd-induced ERS was alleviated when NRK-52E cells were treated with both $\mathrm{Cd}$ and puerarin. Collectively, puerarin was indicated to attenuate ERS following $\mathrm{Cd}$ exposure, via preventing the activation of NRF2 signaling to restore autophagic flux.

ATG7 knockdown has been indicated to result in autophagy deficiency (70) and RAB7 overexpression has been revealed to enhance the fusion of autophagosomes and lysosomes (18). Consequently, both were used in the current 

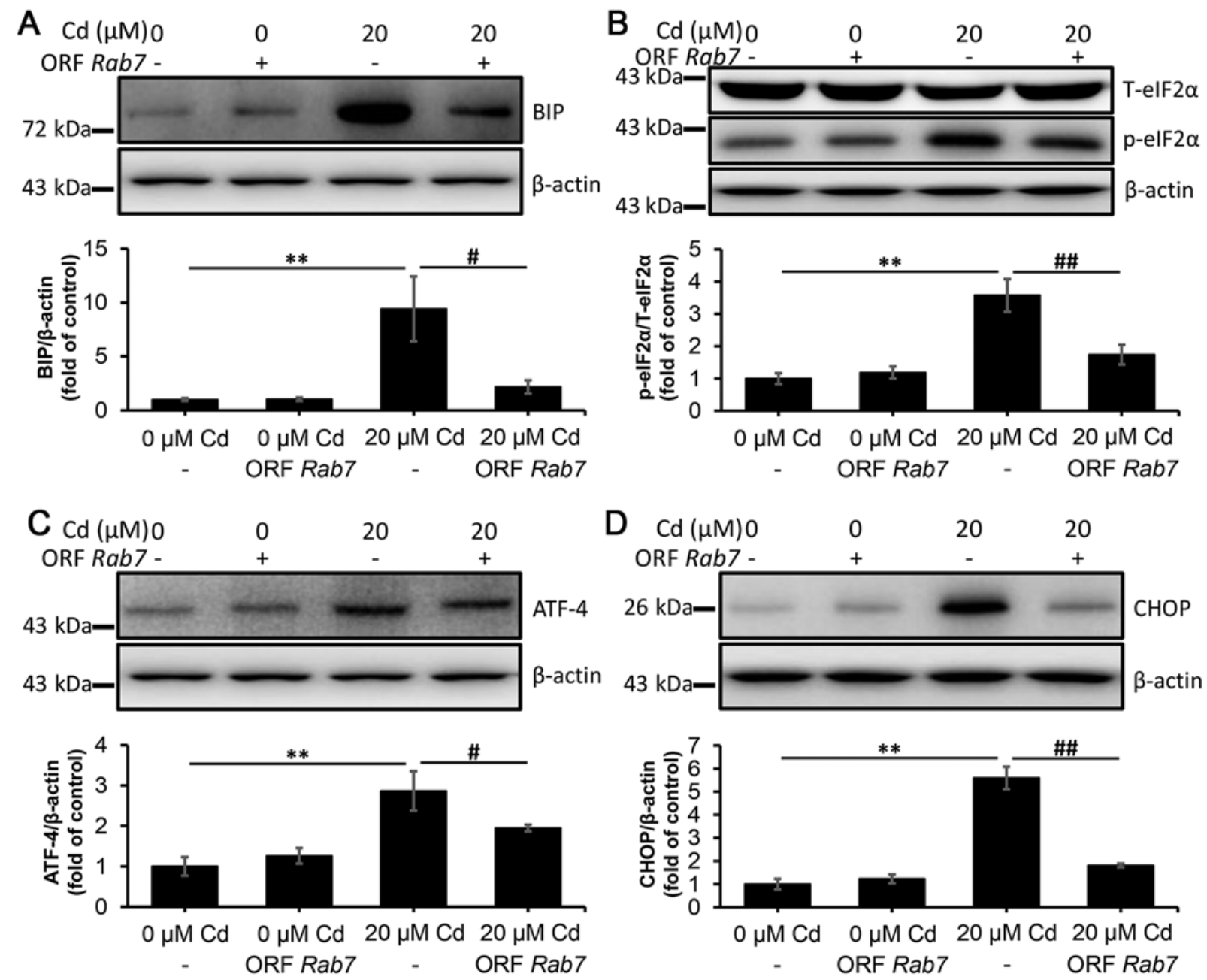

Figure 9. RAB7 overexpression alleviates endoplasmic reticulum stress in NRK-52E cells. The cells were transfected with ORF RAB7 and they were subsequently treated with $20 \mu \mathrm{M} \mathrm{Cd}$ for $12 \mathrm{~h}$. The protein levels of (A) BIP, (B) p-eIF2 $\alpha$, (C) ATF-4 and (D) CHOP were quantified using densitometry following western blotting. $\mathrm{n}=3 .{ }^{* *} \mathrm{P}<0.01$ vs. $0 \mu \mathrm{M} \mathrm{Cd} ;{ }^{*} \mathrm{P}<0.05$ and ${ }^{\# \#} \mathrm{P}<0.01$ vs. $20 \mu \mathrm{M} \mathrm{Cd}$ and puerarin co-treatment. Cd, cadmium; BIP, binding-immunoglobulin protein; p-eIF2 $\alpha$, phosphorylated eukaryotic initiation factor $2 \alpha$; T-eIF2 $\alpha$, total eukaryotic initiation factor $2 \alpha$; ATF-4, activating transcription factor 4 ; CHOP, CCAAT-enhancer-binding protein homologous protein; ORF, open reading frame; RAB7, Ras-related protein Rab-7.

study to further examine the effect of autophagy on ERS. In a previous study, ATG7 gene knockout in a EPCK-Cre Atg7 mouse model has been reported to result in autophagy deficiency, which was indicated by a lack of LC3II and p62/SQSTM1 accumulation (70). In the autophagy deficient cell model of the current study, the Cd-induced upregulation of BIP, p-eIF2 $\alpha$, ATF4 and CHOP was not inhibited by puerarin. This suggested that puerarin alleviated ERS during $\mathrm{Cd}$ exposure primarily via regulating autophagy. As $\mathrm{Cd}$ inhibits, and RAB7 has been indicated to induce, the autolysosome formation (30), the effect of RAB7 was examined in a RAB7 overexpression cell model in the current study. In the overexpressing cells, the Cd-induced increase in ERS markers was attenuated. Moreover, for untreated cells, compared with the empty control vector group, the levels of the ERS markers was slightly increased in the RAB7 overexpression group. This additionally suggested that the autophagic flux serves a critical role in the puerarin-induced alleviation of ERS following treatment with $\mathrm{Cd}$, and excessive autophagy may induce ERS and subsequent cell injury. Autophagy has been revealed to serve a critical role in regulating oxidative stress, and reactive oxygen/nitrogen species may induce ERS and autophagy, indicating that an interaction among oxidative stress, ERS and autophagy exists (26). However, among these three cellular processes, only autophagy has been indicated to exhibit a protective effect against cell damage (71). The results of the present study suggested that the puerarin-mediated restoration of the autophagic flux alleviated $\mathrm{Cd}$-induced ERS. Autophagy is a regulatory mechanism of the cell that removes unnecessary or dysfunctional components, which allows the degradation and recycling of cellular proteins and organelles, especially under stress conditions (11). ERS, which is primarily induced by the accumulation of unfolded or misfolded proteins, is an important factor that promotes cell damage (27). In the present study, the autophagic flux, which was restored via the puerarin-mediated inhibition of the NRF2 signaling pathway, was indicated to promote the degradation of misfolded proteins to alleviate ERS.

Furthermore, ERS can also be activated by oxidative stress, which is induced by ROS, one of its primary sources being dysfunctional organelles, such as mitochondria (27). Therefore, the clearance of dysfunctional organelles to restore the autophagic flux may represent another key mechanism responsible for alleviating ERS during Cd exposure. 
In conclusion, the findings of the present study demonstrated that $\mathrm{Cd}$ induced oxidative stress, apoptosis, activation of NRF2 signaling, obstruction of the autophagic flux and ERS in NRK-52E cells. Moreover, puerarin administration inhibited NRF2 signaling to restore the autophagic flux, via which misfolded proteins and dysfunctional organelles are degraded to alleviate ERS. In the present study, the association between autophagy and ERS was elucidated in the context of $\mathrm{Cd}$-induced renal damage, and it was indicated that puerarin may potentially alleviate environmental $\mathrm{Cd}$-induced nephrotoxicity and improve kidney health in affected populations.

\section{Acknowledgements}

Not applicable.

\section{Funding}

The present study was funded by the National Key Research and Development Program of China (grant no. 2016YFD0501208), the National Natural Science Foundation of China (grant nos. 31101866, 31872533 and 31702305), Jiangsu Provincial Natural Science Foundation of China (grant no. BK20150447), Postdoctoral Research Funding of Yangzhou University (grant no. 137070430) and the Priority Academic Program Development of Jiangsu Higher Education Institutions.

\section{Availability of data and materials}

The datasets used and/or analyzed during the current study are available from the corresponding author on reasonable request.

\section{Authors' contributions}

ZL and GL contributed to the study design and obtained funding. GL and KZ drafted the manuscript. KZ, WD and YT performed the experiments. ML, HZ, WD and YT performed data acquisition, data analysis and interpretation. ML and HZ reviewed the manuscript. All authors read and approved the final manuscript.

\section{Ethics approval and consent to participate}

Not applicable.

\section{Patient consent for publication}

Not applicable.

\section{Competing interests}

The authors declare that they have no competing interests.

\section{References}

1. Jarup L, Rogenfelt A, Elinder CG, Nogawa K and Kjellstrom T: Biological half-time of cadmium in the blood of workers after cessation of exposure. Scand J Work Environ Health 9: 327-331, 1983.

2. Tchounwou PB, Yedjou CG, Patlolla AK and Sutton DJ: Heavy metal toxicity and the environment. Exp Suppl 101: 133-164, 2012.
3. Programme UNE and World Health Organization: Cadmium: Environmental aspects. Environmental Health Criteria, 1992.

4. Lind Y, Engman J, Jorhem L and Glynn AW: Cadmium accumulation in liver and kidney of mice exposed to the same weekly cadmium dose continuously or once a week. Food Chem Toxicol 35: 891-895, 1997.

5. Bernard A: Renal dysfunction induced by cadmium: Biomarkers of critical effects. Biometals 17: 519-523, 2004.

6. Prozialeck WC, Wellington DR, Mosher TL, Lamar PC and Laddaga RA: The cadmium-induced disruption of tight junctions in LLC-PK1 cells does not result from apoptosis. Life Sci 57: 199-204, 1995.

7. Dong Z, Wang L, Xu J, Li Y, Zhang Y, Zhang S and Miao J: Promotion of autophagy and inhibition of apoptosis by low concentrations of cadmium in vascular endothelial cells. Toxicol In Vitro 23: 105-110, 2009.

8. Wang L, Cao J, Chen D, Liu X, Lu H and Liu Z: Role of oxidative stress, apoptosis, and intracellular homeostasis in primary cultures of rat proximal tubular cells exposed to cadmium. Biol Trace Elem Res 127: 53-68, 2009.

9. Shen R, Liu D, Hou C, Liu D, Zhao L, Cheng J, Wang D and Bai D: Protective effect of Potentilla anserina polysaccharide on cadmium-induced nephrotoxicity in vitro and in vivo. Food Funct 8: 3636-3646, 2017.

10. Wang L, Wang H, Li J, Chen D and Liu Z: Simultaneous effects of lead and cadmium on primary cultures of rat proximal tubular cells: Interaction of apoptosis and oxidative stress. Arch Environ Contam Toxicol 61: 500-511, 2011.

11. Mizushima N: Autophagy: Process and function. Genes Dev 21: 2861-2873, 2007.

12. Mizushima N, Yoshimori T and Levine B: Methods in mammalian autophagy research. Cell 140: 313-326, 2010.

13. De Duve C: The lysosome. Sci Am 208: 64-72, 1963.

14. Mizushima N, Levine B, Cuervo AM and Klionsky DJ: Autophagy fights disease through cellular self-digestion. Nature 451: 1069-1075, 2008.

15. Terman A and Brunk UT: Autophagy in cardiac myocyte homeostasis, aging, and pathology. Cardiovasc Res 68: 355-365, 2005.

16. Yang LY, Wu KH, Chiu WT, Wang SH and Shih CM: The cadmium-induced death of mesangial cells results in nephrotoxicity. Autophagy 5: 571-572, 2009.

17. Jin Y, Tanaka A, Choi AM and Ryter SW: Autophagic proteins: New facets of the oxygen paradox. Autophagy 8: 426-428, 2012.

18. Wang Z, Miao G, Xue X, Guo X, Yuan C, Wang Z, Zhang G, Chen Y, Feng D, Hu J and Zhang H: The vici syndrome protein EPG5 Is a Rab7 effector that determines the fusion specificity of autophagosomes with late Endosomes/Lysosomes. Mol Cell 63: 781-795, 2016.

19. Chen M, Li X, Fan R, Yang J, Jin X, Hamid S and Xu S: Cadmium induces BNIP3-dependent autophagy in chicken spleen by modulating miR-33-AMPK axis. Chemosphere 194: 396-402, 2018.

20. Fujishiro H, Liu Y, Ahmadi B and Templeton DM: Protective effect of cadmium-induced autophagy in rat renal mesangial cells. Arch Toxicol 92: 619-631, 2018.

21. So KY, Lee BH and Oh SH: The critical role of autophagy in cadmium-induced immunosuppression regulated by endoplasmic reticulum stress-mediated calpain activation in RAW264.7 mouse monocytes. Toxicology 393: 15-25, 2018.

22. Thevenod F and Lee WK: Cadmium and cellular signaling cascades: Interactions between cell death and survival pathways. Arch Toxicol 87: 1743-1786, 2013.

23. Lee AS: The glucose-regulated proteins: Stress induction and clinical applications. Trends Biochem Sci 26: 504-510, 2001.

24. Kaufman RJ: Orchestrating the unfolded protein response in health and disease. J Clin Invest 110: 1389-1398, 2002.

25. Luo B, Lin Y, Jiang S, Huang L, Yao H, Zhuang Q, Zhao R, Liu H, $\mathrm{He} \mathrm{C}$ and Lin Z: Endoplasmic reticulum stress eIF2 $\alpha-\mathrm{ATF} 4$ pathway-mediated cyclooxygenase-2 induction regulates cadmium-induced autophagy in kidney. Cell Death Dis 7: e2251, 2016.

26. Qi Z and Chen L: Endoplasmic reticulum stress and autophagy. Adv Exp Med Biol 1206: 167-177, 2019.

27. Kang SW and Hegde RS: Lighting up the stressed ER. Cell 135: 787-789, 2008.

28. Oslowski CM and Urano F: Measuring ER stress and the unfolded protein response using mammalian tissue culture system. Methods Enzymol 490: 71-92, 2011.

29. Rivas A, Vidal RL and Hetz C: Targeting the unfolded protein response for disease intervention. Expert Opin Ther Targets 19: 1203-1218, 2015. 
30. Cai Y, Arikkath J, Yang L, Guo ML, Periyasamy P and Buch S: Interplay of endoplasmic reticulum stress and autophagy in neurodegenerative disorders. Autophagy 12: 225-244, 2016.

31. Khaminets A, Heinrich T, Mari M, Grumati P, Huebner AK, Akutsu M, Liebmann L, Stolz A, Nietzsche S, Koch N, et al Regulation of endoplasmic reticulum turnover by selective autophagy. Nature 522: 354-358, 2015.

32. Zhang L, Xia Q, Zhou Y and Li J: Endoplasmic reticulum stress and autophagy contribute to cadmium-induced cytotoxicity in retinal pigment epithelial cells. Toxicol Lett 311: 105-113, 2019.

33. Hernandez-Gea V, Hilscher M, Rozenfeld R, Lim MP, Nieto N, Werner S, Devi LA and Friedman SL: Endoplasmic reticulum stress induces fibrogenic activity in hepatic stellate cells through autophagy. J Hepatol 59: 98-104, 2013.

34. Digaleh H, Kiaei M and Khodagholi F: Nrf2 and Nrf1 signaling and ER stress crosstalk: Implication for proteasomal degradation and autophagy. Cell Mol Life Sci 70: 4681-4694, 2013.

35. Bellezza I, Giambanco I, Minelli A and Donato R: Nrf2-Keap1 signaling in oxidative and reductive stress. Biochim Biophys Acta Mol Cell Res 1865: 721-733, 2018.

36. Tang Z, Hu B, Zang F, Wang J, Zhang X and Chen H: Nrf2 drives oxidative stress-induced autophagy in nucleus pulposus cells via a Keap1/Nrf2/p62 feedback loop to protect intervertebral disc from degeneration. Cell Death Dis 10: 510, 2019.

37. Dodson M, Redmann M, Rajasekaran NS, Darley-Usmar V and Zhang J: KEAP1-NRF2 signalling and autophagy in protection against oxidative and reductive proteotoxicity. Biochem J 469: 347-355, 2015

38. Ichimura Y, Waguri S, Sou YS, Kageyama S, Hasegawa J, Ishimura R, Saito T, Yang Y, Kouno T, Fukutomi T, et al: Phosphorylation of $\mathrm{p} 62$ activates the Keap1-Nrf2 pathway during selective autophagy. Mol Cell 51: 618-631, 2013.

39. Li W, Yang Q and Mao Z: Signaling and induction of chaperone-mediated autophagy by the endoplasmic reticulum under stress conditions. Autophagy 14: 1094-1096, 2018.

40. Ryabaya O, Prokofieva A, Khochenkov D, Abramov I, Zasedatelev A and Stepanova E: Inhibition of endoplasmic reticulum stress-induced autophagy sensitizes melanoma cells to temozolomide treatment. Oncol Rep 40: 385-394, 2018.

41. Liu CM, Ma JQ, Liu SS, Feng ZJ and Wang AM: Puerarin protects mouse liver against nickel-induced oxidative stress and inflammation associated with the TLR4/p38/CREB pathway. Chem Biol Interact 243: 29-34, 2016.

42. Wang N, Zhang Y, Wu L, Wang Y, Cao Y, He L, Li X and Zhao J Puerarin protected the brain from cerebral ischemia injury via astrocyte apoptosis inhibition. Neuropharmacology 79: 282-289, 2014.

43. Wang B, Chen S, Yan X, Li M, Li D, Lv P and Ti G: The therapeutic effect and possible harm of puerarin for treatment of stage III diabetic nephropathy: A meta-analysis. Altern Ther Health Med 21: 36-44, 2015.

44. Shapiro DL: Morphological and biochemical alterations in foetal rat brain cells cultured in the presence of monobutyryl cyclic AMP. Nature 241: 203-204, 1973.

45. Lin YN, Jiang M, Chen WJ, Zhao TJ and Wei YF: Cancer and ER stress: Mutual crosstalk between autophagy, oxidative stress and inflammatory response. Biomed Pharmacother 118: 109249, 2019.

46. Klionsky DJ, Abdelmohsen K, Abe A, Abedin MJ, Abeliovich H, Acevedo Arozena A, Adachi H, Adams CM, Adams PD, Adeli K, et al: Guidelines for the use and interpretation of assays for monitoring autophagy (3rd edition). Autophagy 12: 1-222, 2016

47. Zhou XL, Wan XM, Fu XX and Xie CG: Puerarin prevent cadmium-induced hepatic cell damage by suppressing apoptosis and restoring autophagic flux. Biomed Pharmacother 115 108929, 2019.

48. Cybulsky AV: Endoplasmic reticulum stress, the unfolded protein response and autophagy in kidney diseases. Nat Rev Nephrol 13 681-696, 2017.

49. Yu SN, Kim SH, Kim KY, Ji JH, Seo YK, Yu HS and Ahn SC: Salinomycin induces endoplasmic reticulum stressmediated autophagy and apoptosis through generation of reactive oxygen species in human glioma U87MG cells. Oncol Rep 37: 3321-3328, 2017.

50. Duann P, Lianos EA, Ma J and Lin PH: Autophagy, innate immunity and tissue repair in acute kidney injury. Int J Mol Sci 17: $662,2016$.
51. Coker-Gurkan A, Ayhan-Sahin B,KecelogluG,Obakan-Yerlikaya P, Arisan ED and Palavan-Unsal N: Atiprimod induce apoptosis in pituitary adenoma: Endoplasmic reticulum stress and autophagy pathways. J Cell Biochem 120: 19749-19763, 2019.

52. Chou X, Ding F, Zhang XY, Ding XQ, Gao H and Wu Q: Sirtuin-1 ameliorates cadmium-induced endoplasmic reticulum stress and pyroptosis through XBP-1s deacetylation in human renal tubular epithelial cells. Arch Toxicol 93: 965-986, 2019.

53. Wang JC, Zhu HL, Zhang C, Wang HW and Yang ZJ: Baicalein ameliorates cadmium-induced hepatic and renal oxidative damage in rats. Indian J Anim Res 53: 523-527, 2019.

54. NairAR,LeeWK, Smeets K, SwennenQ, SanchezA, Thévenod Fand Cuypers A: Glutathione and mitochondria determine acute defense responses and adaptive processes in cadmium-induced oxidative stress and toxicity of the kidney. Arch Toxicol 89: 2273-2289, 2015.

55. Pathak N and Khandelwal S: Role of oxidative stress and apoptosis in cadmium induced thymic atrophy and splenomegaly in mice. Toxicol Lett 169: 95-108, 2007.

56. Chen $\mathrm{J}$ and Shaikh ZA: Activation of Nrf2 by cadmium and its role in protection against cadmium-induced apoptosis in rat kidney cells. Toxicol Appl Pharmacol 241: 81-89, 2009.

57. Godt J, Scheidig F, Grosse-Siestrup C, Esche V, Brandenburg P, Reich A and Groneberg DA: The toxicity of cadmium and resulting hazards for human health. J Occup Med Toxicol 1: 22, 2006.

58. Alli LA: Blood level of cadmium and lead in occupationally exposed persons in Gwagwalada, Abuja, Nigeria. Interdiscip Toxicol 8: 146-150, 2015.

59. Goyal T, Mitra P, Singh P, Sharma S and Sharma P: Assessement of blood lead and cadmium levels in occupationally exposed workers of Jodhpur, Rajasthan. Ind J Clin Biochem, 2020 https://doi.org/10.1007/s12291-020-00878-6.

60. Gu J, Dai SY, Liu YM, Liu H, Zhang Y, Ji X, Yu F, Zhou Y, Chen L, Tse WKF, et al: Activation of $\mathrm{Ca}(2+)$-sensing receptor as a protective pathway to reduce Cadmium-induced cytotoxicity in renal proximal tubular cells. Sci Rep 8: 1092, 2018.

61. Tu W, Wang H, Li S, Liu Q and Sha H: The anti-inflammatory and anti-oxidant mechanisms of the Keap1/Nrf2/ARE signaling pathway in chronic diseases. Aging Dis 10: 637-651, 2019.

62. Chen DL, Tavana O, Chu B, Erber L, Chen Y, Baer R and Gu W: NRF2 is a major target of ARF in p53-independent tumor suppression. Mol Cell 68: 224-232.e4, 2017.

63. Towers CG, Fitzwalter BE, Regan D, Goodspeed A, Morgan MJ, Liu CW, Gustafson DL and Thorburn A: Cancer cells upregulate NRF2 signaling to adapt to autophagy inhibition. Dev Cell 50: 690-703.e6, 2019.

64. Guerrero-Hue M, Farre-Alins V, Palomino-Antolin A, Parada E, Rubio-Navarro A, Egido J, Egea J and Moreno JA: Targeting Nrf2 in protection against renal disease. Curr Med Chem 24: 3583-3605, 2017

65. Ma JQ, Ding J, Xiao ZH and Liu CM: Puerarin ameliorates carbon tetrachloride-induced oxidative DNA damage and inflammation in mouse kidney through ERK/Nrf2/ARE pathway. Food Chem Toxicol 71: 264-271, 2014

66. Ullah MZ, Khan AU, Afridi R, Rasheed H, Khalid S, Naveed M, Ali H, Kim YS and Khan S: Attenuation of inflammatory pain by puerarin in animal model of inflammation through inhibition of pro-inflammatory mediators. Int Immunopharmacol 61: 306-316, 2018.

67. Chen ZJ, Chen JX, Wu LK, Li BY, Tian YF, Xian M, Huang ZP and Yu RA: Induction of endoplasmic reticulum stress by cadmium and its regulation on Nrf2 signaling pathway in kidneys of rats. Biomed Environ Sci 32: 1-10, 2019.

68. Liao W, Wang Z, Fu Z, Ma H, Jiang M, Xu A and Zhang W: p62/SQSTM1 protects against cisplatin-induced oxidative stress in kidneys by mediating the cross talk between autophagy and the Keap1-Nrf2 signalling pathway. Free Radic Res 53: 800-814, 2019.

69. Manley S, Ni HM, Kong B, Apte U, Guo G and Ding WX: Suppression of autophagic flux by bile acids in hepatocytes. Toxicol Sci 137: 478-490, 2014.

70. Khambu B, Huda N, Chen X, Antoine DJ, Li Y, Dai G, Köhler UA, Zong WX, Waguri S, Werner S, et al: HMGB1 promotes ductular reaction and tumorigenesis in autophagy-deficient livers. J Clin Invest 129: 2163, 2019.

71. Nakka VP, Prakash-Babu P and Vemuganti R: Crosstalk between endoplasmic reticulum stress, oxidative stress, and autophagy: Potential therapeutic targets for acute CNS injuries. Mol Neurobiol 53: 532-544, 2016. 